\title{
The Different Strategies Used by Salmonella to Invade Host Cells
}

\author{
Rosselin Manon, Abed Nadia, Namdari Fatémeh, \\ Virlogeux-Payant Isabelle, Velge Philippe and Wiedemann Agnès \\ INRA Centre de Tours, UR1282 Infectiologie Animale et Santé Publique, Nouzilly \\ IFR 136, Agents Transmissibles et Infectiologie, Nouzilly
}

France

\section{Introduction}

Salmonella enterica are members of a Gram-negative enteropathogenic bacteria family, which are able to infect a great diversity of hosts, including human. According to serotypes and hosts, Salmonella enterica cause a wide range of food- and water-borne diseases ranging from self-limiting gastroenteritis to systemic typhoid fever. Moreover, no other known bacterial pathogens belonging to a single species show such a remarkable variation in their host specificity. Ubiquitous serotypes such as Typhimurium and Enteritidis tend to produce acute but self-limiting enteritis in a wide range of hosts, whereas host-specific serotypes are associated with severe systemic disease in healthy outbred adults of a single species that may not involve diarrhoea (e.g. Gallinarum in poultry). Host-restricted serotypes are primarily associated with systemic disease in one host (e.g. Dublin in cattle, Choleraesuis in pigs), but may cause disease in a limited number of other species (Velge et al., 2005).

For all these serotypes, the intestinal barrier crossing constitutes a crucial step for infection establishment. As shown in Figure 1, Salmonella can induce their own entry into enterocytes, but $\mathrm{M}$ cells and CD18-expressing phagocytes also facilitate their translocation through the intestinal epithelium (Watson \& Holden, 2010). During gastroenteritis pathology, host colonization is restricted to the intestinal tract. However, Salmonella also have the ability to disseminate to extra-intestinal sites at least via CD18-expressing phagocytes, leading to deep organ colonization (Vazquez-Torres et al., 1999).

Bacterial pathogens have developed two different mechanisms to invade non-phagocytic host cells by hijacking physiological cellular processes. Bacteria, such as Listeria monocytogenes and Yersinia pseudotuberculosis express surface proteins that interact with receptor on the host cell plasma membrane. This interaction promotes an activation of host cell signaling pathways, leading to actin remodelling. This process is referred to as a Zipper mechanism and is characterized by the induction of little protrusive activity and thin membrane extensions (Figure 2A and C) (Cossart \& Sansonetti, 2004). Other bacteria, such as Shigella flexneri, do not require a receptor but trigger internalization from "inside" via the action of pathogen-effector proteins delivered by specialized protein secretion systems (Schroeder \& Hilbi, 2008). Translocated effector proteins effectively allow the bacteria to "hijack" many essential 
intracellular processes and induce a massive reorganization of the host actin cytoskeleton, resulting in intense membrane ruffling and internalization of the bacteria. This invasion process is referred to as a Trigger mechanism (Figure 2B and D) (Cossart \& Sansonetti, 2004).

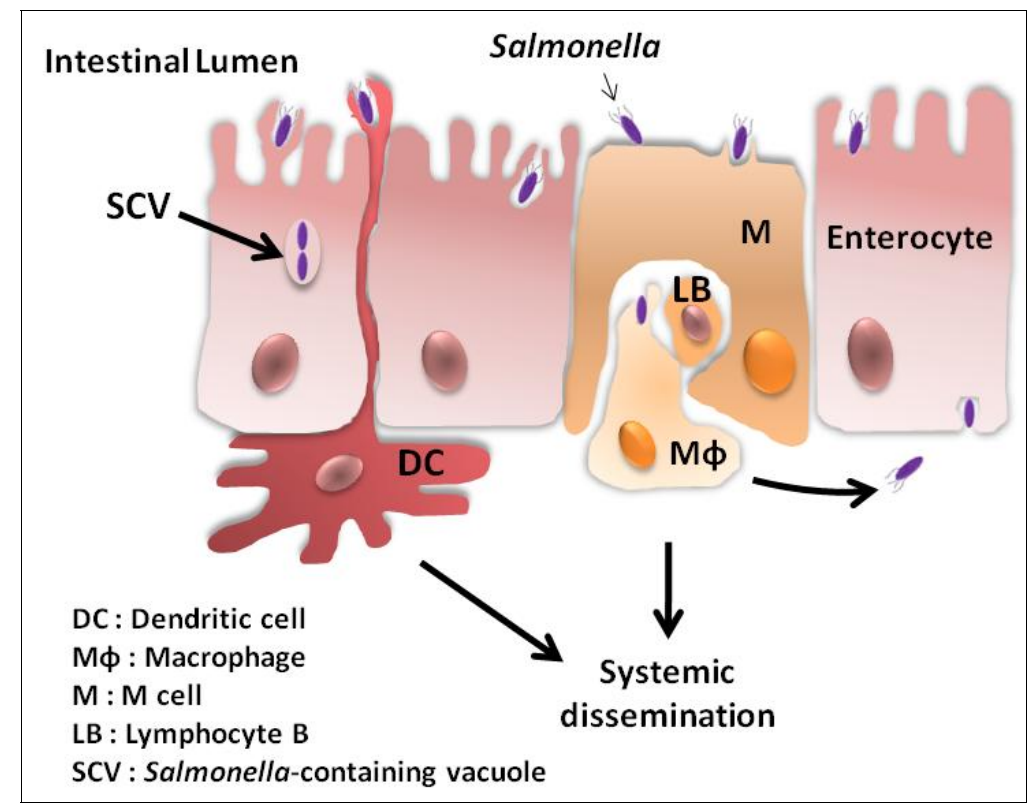

Fig. 1. Intestinal barrier crossing by Salmonella enterica through M cells, enterocytes or following a luminal capture by CD18+ phagocytes such as dendritic cells.

The reorganization of actin cytoskeleton at the entry site is a crucial step for Trigger and Zipper bacterial internalization. In eukaryotic cells, actin exists as a globular monomer (Gactin) which can assemble to form a filamentous structure (F-actin). In physiological conditions, actin polymerization requires different steps. First, nucleation of actin which consists in regrouping three actin monomers, is stimulated by cellular factors such as the Arp2/3 complex (Mullins et al., 1998). Once nucleated, the addition of ATP-actin-monomers at the barbed extremity of the filaments allows actin elongation (Pollard et al., 2000). The three-dimensional structure of actin filaments is ensured by capping proteins and other actin-binding proteins such as actinin, gelsolin, and villin that enable bundling of filaments (Bretscher, 1991; Hartwig \& Kwiatkowski, 1991). Actin dynamics regulation is closely associated with small Rho guanosine triphosphatase protein (RhoGTPase) activity. RhoGTPases cycle between an inactive guanine di-phosphate (GDP)-bound form and an active guanine tri-phosphate (GTP)-bound form. The switch between inactive and active state is regulated by guanine exchange factors (GEF) which catalyze the exchange of GDP with GTP and GTPase activating proteins (GAP) which hydrolyze GTP into GDP to switch off their active state. When bound to GTP, Rho GTPases target and activate downstream effectors such as proteins from the Wiscott-Aldrich Syndrome protein (WASP) / N-WASP Family, leading to nucleator activation and actin reorganization. All these steps are required during bacterial internalization. 


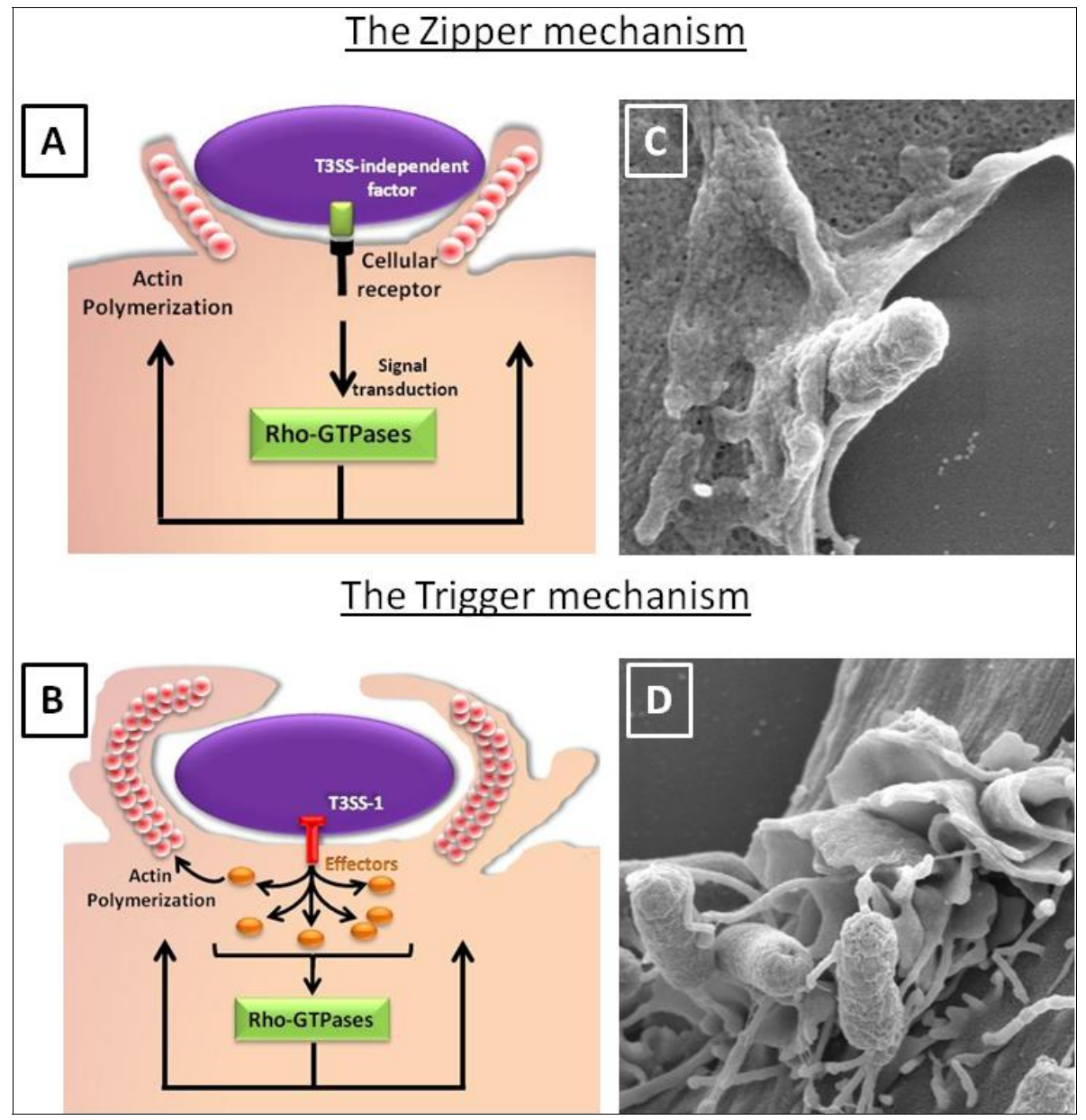

Fig. 2. Models of Zipper and Trigger invasion mechanisms. (A) The Zipper process is initiated by an interaction between a host cell receptor and a bacterial surface protein which allows the activation of RhoGTPases and actin polymerization at the entry site. (B) In contrast, during the Trigger mechanism, RhoGTPases are targeted by bacterial effectors which are directly translocated into host cell via a type-three secretion system, leading to actin polymerization and internalization. Electron scanning microscopy pictures show (C) $S$. Enteritidis invading fibroblasts via a Zipper process which is characterized by weak membrane rearrangements and (D) via a Trigger process which is characterized by intense membrane rearrangements.

The study of host cell invasion by Salmonella has been initiated in 1967 by Takeuchi (Takeuchi, 1967). For decades, it was described in the literature that Salmonella can enter cells only via a "Trigger" mechanism mediated by a type-three secretion system (T3SS-1) encoded by the Salmonella pathogenicity island-1 (SPI-1) (Ibarra \& Steele-Mortimer, 2009). Recent data have showed that cell invasion could occur despite the absence of the T3SS-1 
(Aiastui et al., 2010; Radtke et al., 2010; Rosselin et al., 2011), indicating that the dominant paradigm postulating that a functional SPI-1/T3SS is absolutely required for cell entry, should be reconsidered. Moreover, the characterization of one T3SS-1-independent invasion pathway revealed that Salmonella have also the ability to enter cells via a Zipper process mediated by the Rck invasin (Rosselin et al., 2011). Consequently Salmonella are the first bacteria found to be able to invade cells both via a Zipper and a Trigger mechanism.

Here, our current understanding of the different strategies used by Salmonella to invade host cells will be summarized and we will focus on how Salmonella are able to manipulate the host actin cytoskeleton, leading to discrete or intense membrane rearrangements. The gap of our knowledge about these different entry pathways will be discussed.

\section{Invasion mechanism dependent on the T3SS-1}

The Type-Three Secretion System (T3SS-1) is the best characterized invasion system of Salmonella. It allows bacterial internalization into non-phagocytic cells via a Trigger mechanism which induces massive actin rearrangements and intense membrane ruffling at the entry site (Cossart \& Sansonetti, 2004). Under environmental conditions that enable the expression of the T3SS-1, the secretion apparatus is assembled at the bacterial surface and effectors are translocated into the eukaryotic cytosol following an interaction between the bacteria and the host cell (Garner et al., 2002; Hayward et al., 2005).

\subsection{T3SS-1 structure}

T3SSs are supramolecular complexes that play a major role in the virulence of many Gramnegative pathogens by injecting bacterial protein effectors directly into host cells in an energy-dependent (ATP) manner (Galan \& Wolf-Watz, 2006). These complexes cross both inner and outer membranes of bacteria and are able to create a pore in eukaryotic membrane upon contact with a host cell. They are made of an exportation apparatus, a basal body, a needle and a translocon at the tip of the needle (Figure 3A). The structure of these T3SSs shows a high degree of conservation among pathogens (Tampakaki et al., 2004) and the Salmonella T3SS-1 apparatus shares in particular a high homology with the T3SS of Shigella, also involved in host cell invasion (Groisman \& Ochman, 1993).

The basal body of the T3SS anchors the complex into the bacterial inner and outer membranes (Figure 3A). It is composed of PrgH, PrgK and InvG proteins which assemble into an inner ring (PrgH and PrgK) and an outer ring (InvG) (Schraidt \& Marlovits, 2011). Anchored to the basal body via its transmembrane part, the needle protrudes from the outer membrane as a long filament of $50 \mathrm{~nm}$ length and is composed of the single PrgI protein (Kimbrough \& Miller, 2000). At the extremity of the needle, a complex of three proteins (SipB, SipC, SipD), known as the translocon, is able to form a pore in the eukaryotic target cell, allowing the secretion of effector proteins (Mattei et al., 2011). SipB, SipC and SipD proteins (also referred to Ssp proteins) share homology with other translocon proteins such as IpaB, IpaC and IpaD proteins of Shigella (Hueck, 1998). SipD has a hydrophilic domain and interacts directly with the PrgI needle protein (Rathinavelan et al., 2011) while the two other proteins of the translocon (SipB and SipC) have a hydrophobic domain and are therefore directly involved in the pore formation (Hayward et al., 2000; Miki et al., 2004). Particularly, it has been shown that the interaction of SipB with cellular cholesterol is necessary for effector translocation (Hayward et 
al., 2005). Finally, the translocation of T3SS-1 effector proteins requires an exportation apparatus located at the inner membrane level and made of highly conserved proteins among T3SSs (SpaP, SpaQ, SpaR, SpaS, InvA, InvC and OrgB). The unfolded effectors in association with their chaperone are targeted to the exportation apparatus and the ATPase InvC produces the energy necessary to the transport of these proteins through the needle (Akeda \& Galan, 2004). The appropriate hierarchy in the secretion process is established by a cytoplasmic sorting platform composed of SpaO, OrgA and OrgB (Lara-Tejero et al., 2011). This platform sequentially loads the secreted proteins by interacting with their chaperones to ensure a specific order of secretion and optimize host cell invasion.

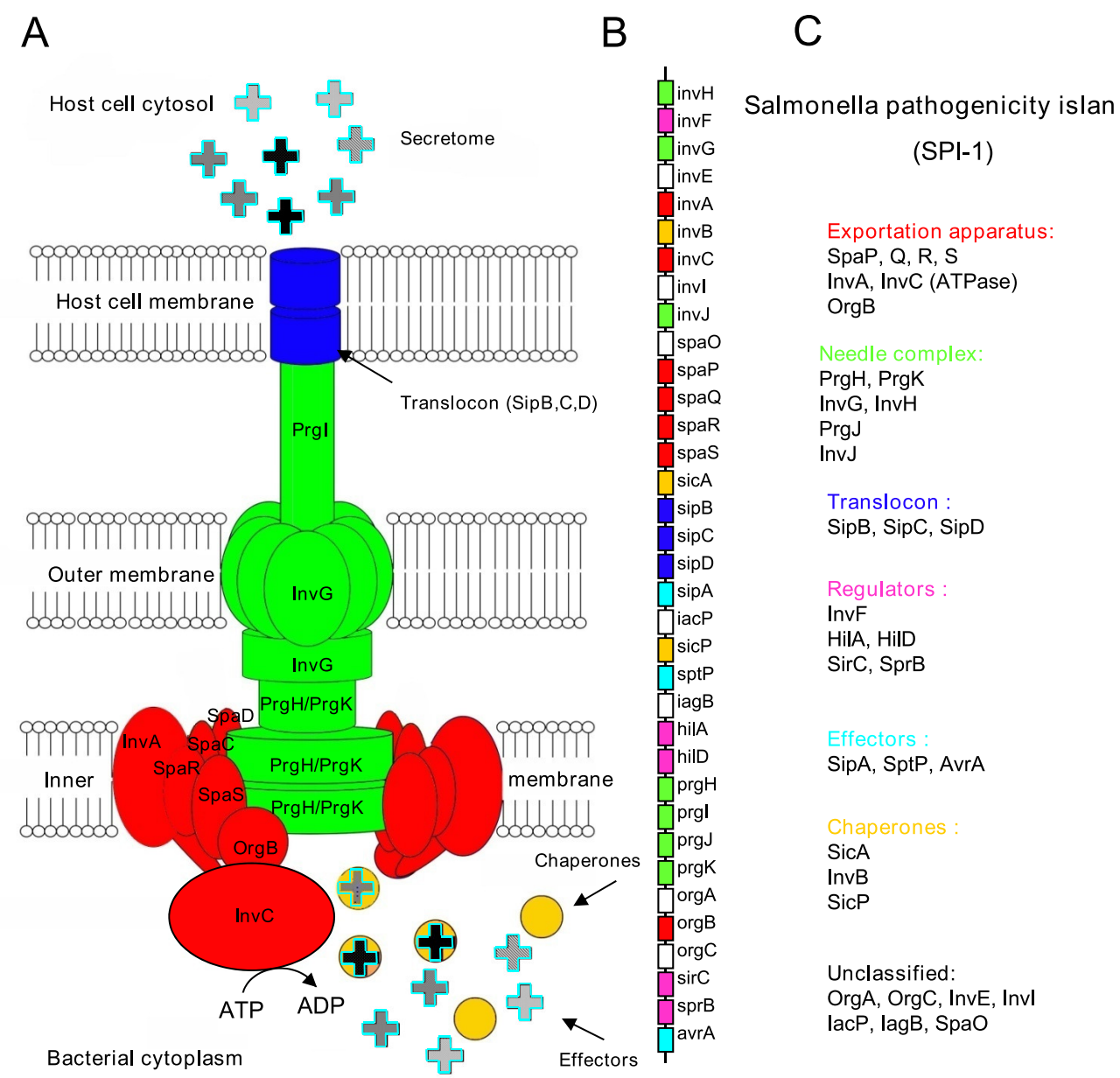

Adapted from Kimbrough and Miller, 2002.

A. Localization of the T3SS-1 structure proteins. B. Schematic representation of SPI-1 island encoding the T3SS-1 proteins. C. Functional classification of SPI-1-encoded proteins.

Fig. 3. Structure and organization of Salmonella T3SS-1. 


\subsection{Regulation of T3SS-1 expression}

During Salmonella infection, a crucial step is the crossing of the intestinal barrier. The host environment encountered by the bacteria, and more particularly the small intestine environment, plays a major role in the invasion as it controls expression of the secretion apparatus. Coordination of T3SS- 1 expression genes, almost all located on the Salmonella Pathogenicity Island 1 (SPI-1) is complex and well-timed. In response to different environmental stimuli, a sophisticated regulatory network controlling the expression of SPI1 has been established (Figure 4). Our purpose here is not to set up the thorough state-ofthe-art on all the regulators involved in Salmonella invasion, but to give a general overview of this system (for a review, see (Ellermeier \& Slauch, 2007)).

SPI-1 contains 39 genes encoding structural T3SS-1 proteins (inv/spa and prg operon), translocon proteins (SipB, C, D), some effectors (SipA, SptP and AvrA), some chaperones (SicA, InvB, SicP) and finally four transcriptional regulators (HilA, hilC, HilD and InvF) (Figure 3B and C). Other genes encoding secreted effectors ( $\operatorname{sop} A, \operatorname{sop} B, \operatorname{sopD}, \operatorname{sop} E$, sopE2, $s l r P, s s p H 1, s s p H 2)$ are located elsewhere on the chromosome.

HilA is central for SPI-1 transcriptional regulation. This protein activates directly the transcription of $p r g$, inv/spa and sip operons, encoding structural components and some secreted effectors of T3SS-1 respectively. In addition, HilA induces the transcription of invF, encoding a transcriptional activator and targeting, among others, sip operon, $\operatorname{sop} E$ and $\operatorname{sop} B$ genes (Darwin \& Miller, 1999). The sequential expression of HilA and InvF regulators allows a hierarchical regulation of invasion genes.

Then, a second crucial level of SPI-1 transcriptional regulation takes place through the regulation of HilA via a feed-forward loop, involving three homologous transcriptional regulators: HilC, HilD and RtsA. Each of them binds directly to the hilA promoter and is able to activate its own expression. In fact, HilC, HilD and probably RtsA, act as derepressors of hilA transcription by counteracting the silencing exerted by nucleoidstructuring proteins such as H-NS or Hha (Akbar et al., 2003; Queiroz et al., 2011). The reason why HilC, HilD and RtsA play such an important role in T3SS-1 expression through hilA regulation is that they are at the integration point of a lot of signals that control SPI-1 expression (Figure 4). In this regulatory circuit, it is currently admitted that HilD has a predominant role whereas HilC and RtsA simply act as signal amplifiers. However, it has also been shown that these three regulators are also directly implicated in the regulation of others invasion genes (Akbar et al., 2003; Ellermeier \& Slauch, 2004).

Moreover, besides these direct regulators, a great number of other hilA regulators, acting mainly through HilD, have been identified. Among them, two-component systems play a major role. They sense environmental conditions and allow the transmission of different signals which modulate T3SS-1 genes expression. Some are able to activate indirectly HilA expression such as BarA/SirA and OmpR/EnvZ, whereas others such as PhoP/PhoQ and PhoB/PhoR repress it. In fact, HilA expression can also be inhibited. HilE has been identified as a negative regulator of hilA transcription preventing HilD activity (Fahlen et al., 2000; Baxter et al., 2003). It has been suggested that the two-component systems PhoP/PhoQ and PhoB/PhoR act through HilE to regulate SPI-1 (Baxter \& Jones, 2005).

As stated above, environmental signals play a major role in Salmonella invasion. Low oxygen tension, high osmolarity, high iron concentration, neutral $\mathrm{pH}$ are conditions found in the 
ileum, known to be the preferential invasion site of Salmonella. Thus, as expected in these conditions, invasion genes are activated through hilA expression. In contrast, when Salmonella are located at unfavorable sites for invasion in the host organism, the presence of signals such as bile, secreted into the proximal small intestine or cationic peptides, known to exist in macrophages, inhibits T3SS-1 expression (Figure 4).

Although much has already been identified about the regulation of SPI-1, recently, it became more evident that mechanisms regulating this system are more complex than previously thought.

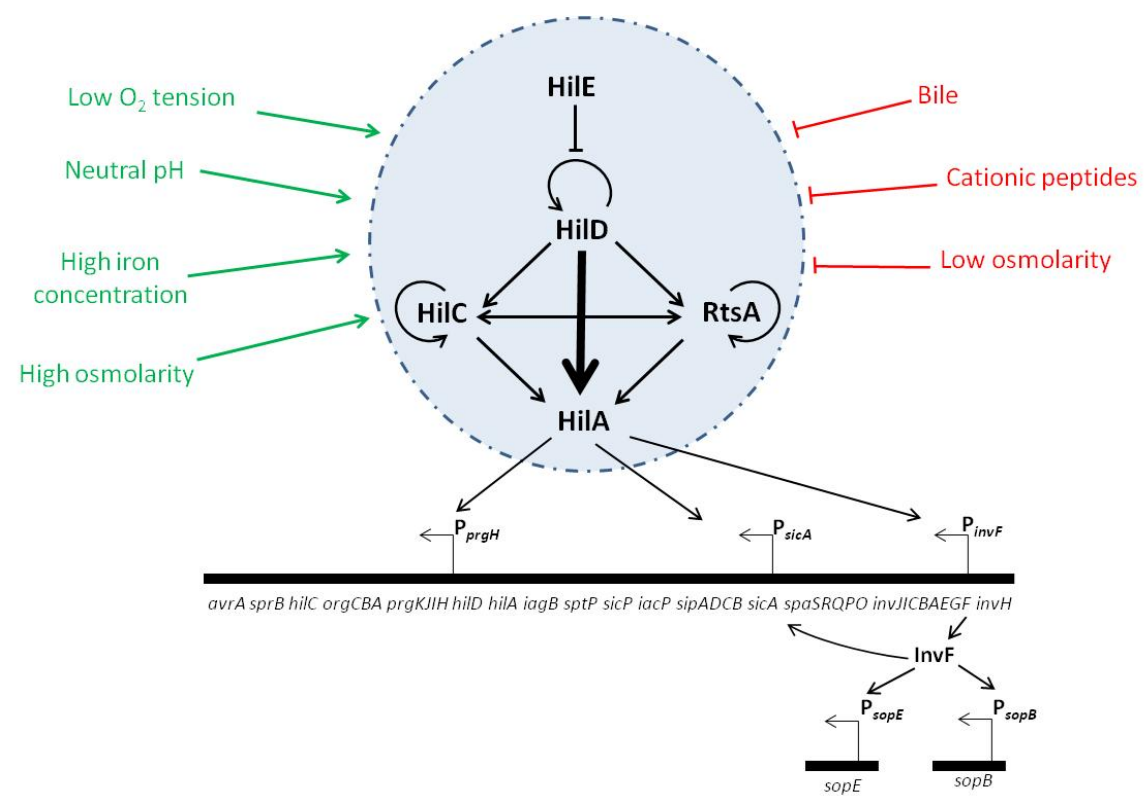

Fig. 4. Environmental and transcriptional regulation of SPI-1 encoded genes. HilA is the major regulator of SPI-1 and is itself regulated by other regulators such as HilC, HilD, RtsA and HilE. When Salmonella reach the small intestine, a low $\mathrm{O}_{2}$ tension, a neutral $\mathrm{pH}$, a high iron concentration and a high osmolarity activate SPI-1 expression. In contrast, the presence of bile or cationic peptides represses its expression.

\subsection{Subversion of the cellular machinery during T3SS-1-dependent entry}

Among the effectors that are translocated into host cell by the T3SS-1, six are essential to cell invasion (SipA, SipC, SopB, SopD, SopE, SopE2) while the other effectors contribute to a variety of post-invasion processes such as host cell survival and modulation of the inflammatory response (Patel \& Galan, 2005). To trigger internalization into cells, effectors manipulate actin cytoskeleton either directly or indirectly. They also manipulate the delivery of vesicles to the site of bacterial entry to provide additional membrane and allow the extension and ruffling of the plasma membrane necessary to promote invasion. In later steps, membrane fission occurs to induce the sealing of the future Salmonellacontaining vacuole (SCV) and actin filaments are depolymerized, enabling the host cell to recover its normal shape (Figure 5). 


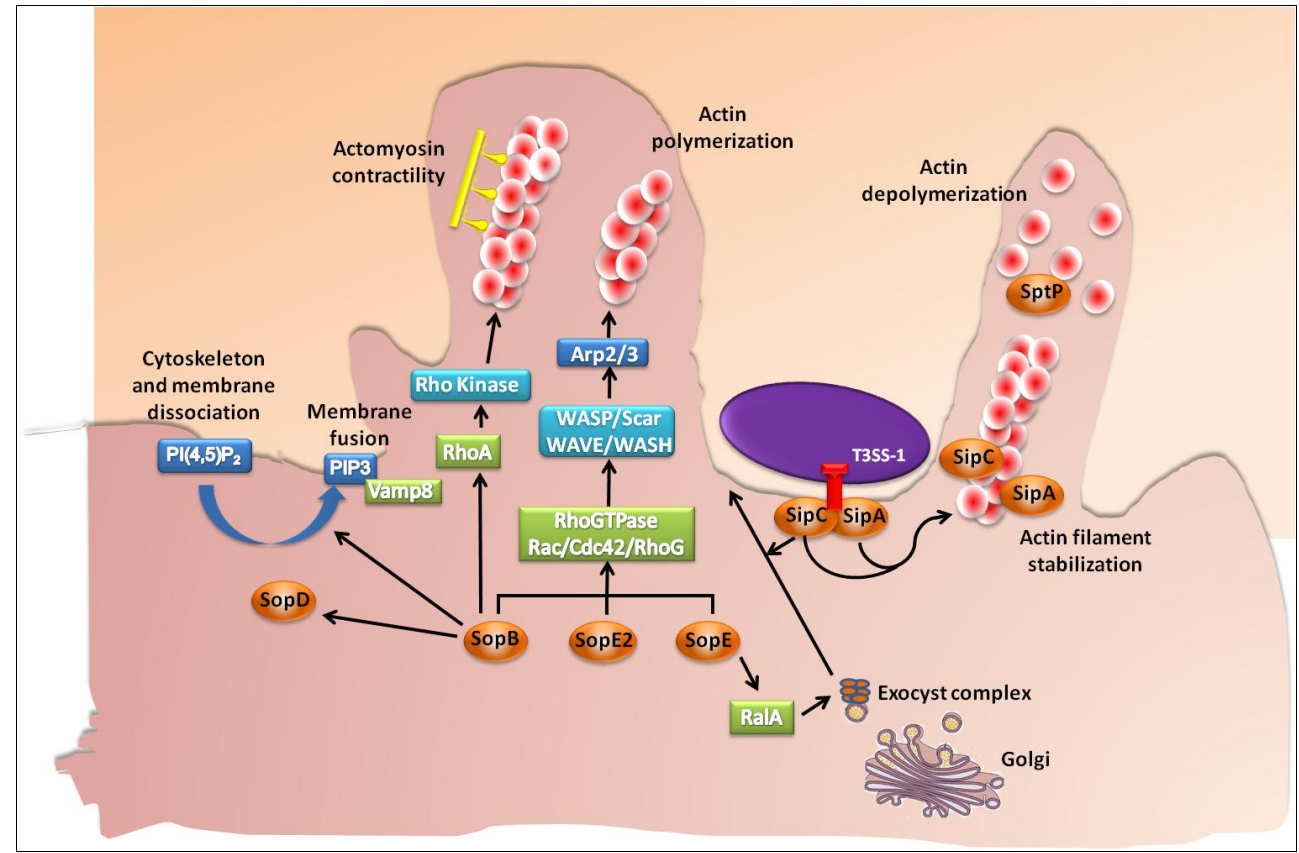

Fig. 5. Intracellular activities of the T3SS-1 effectors: SipA and SipC bind actin directly whereas SopE, SopE2 and SopB stimulate RhoGTPase activity. SipC and SopE also act in cooperation to recruit exocytic vesicles at the entry site. SopB plays diverse roles during invasion: it promotes actomyosin contractility and changes phosphoinositide concentrations to facilitate the dissociation between actin cytoskeleton and membrane at the entry site. SopB also probably triggers the delivery of vesicles to the bacterial entry site in a VAMP-8 dependent way and activate SopD which seems to contribute to the sealing and the formation of the SCV. The last effector SptP turns off the activity of RhoGTPases to allow the host cell to regain its initial shape.

\subsubsection{Actin manipulation}

Actin polymerization is an essential process induced by T3SS- 1 effectors during entry. Some effectors stimulate actin rearrangements indirectly and two effectors, SipA and SipC, manipulate actin directly. SipA and SipC are localized at the eukaryotic plasma membrane during entry (Scherer et al., 2000) but they are also translocated into the host cell cytoplasm (Hueck et al., 1995; Kaniga et al., 1995).

SipC, as part of the translocon, is required for T3SS-1 effector translocation and involved in actin nucleation and bundling. Due to its various functions, a 95\% decrease in invasion is observed when sipC is deleted (Chang et al., 2005). The C-terminal region of SipC (aminoacids A321-A409) encodes the effector translocation activity (Chang et al., 2005) and its central region (amino-acids N201-S220) binds to actin and induces a rapid nucleation and elongation of actin filaments. More precisely the regions containing the amino acids from H221-M260 and L381-A409 bind to and bundle actin filaments in vitro (Myeni \& Zhou, 
2010). The bundling activity of SipC is essential for internalization as a sipC mutant lacking bundling activity is impaired in cell invasion. SipC dimerization / multimerization seems to be required for nucleation and a $\operatorname{sip} C$ mutant deficient for multimerization and actin nucleation failed to cause severe colitis in a mouse model (Chang et al., 2007).

As SipC, SipA binds directly to actin (amino acids P446-R685) and modulates cytoskeleton dynamics in different ways (Galkin et al., 2002; Higashide et al., 2002). A Salmonella Typhimurium sipA mutant exhibits a $60-80 \%$ decrease in invasion compared to the wildtype strain (Perrett \& Jepson, 2009), which can be correlated to the fact that SipA is a multifunctional effector. First, Zhou et al. (1999b) have observed that SipA reduces the critical concentration of G-actin in the cytosol required for polymerization. In addition, to facilitate polymerization, SipA also stabilizes actin filaments by displacing ADF / cofilin factor which stimulates actin depolymerization and by protecting F-actin from gelsolin severing (McGhie et al., 2004). Moreover, SipA enhances actin cross-linking both by interacting with T-Plastin, a cellular bundling protein, and by enhancing SipC activity (Zhou et al., 1999a; McGhie et al., 2001). Interestingly, Perrett and Jepson have demonstrated that a sipA deletion induces a decrease in host cell invasion but without affecting the frequency of membrane ruffle formation (Perrett \& Jepson, 2009). By visualizing the membrane ruffles in the absence of SipA, they have observed that SipA is required to ensure the spatial localization of actin rearrangement beneath invading Salmonella for efficient uptake of bacteria.

In contrast to SipA and SipC, SopB, SopE and SopE2 exert their activity into host cells by inducing actin polymerization in an indirect way. They activate RhoGTPases which are key cellular effectors that regulate actin cytoskeleton remodeling (Patel \& Galan, 2005).

During Salmonella T3SS-1 dependent invasion, SopE and SopE2 effectors mimic GEF activity to activate RhoGTPases. Their activation induces actin polymerization by stimulating downstream cellular proteins such as N-WASP, WAVE and WASH which activate the Arp2/3 nucleator complex (Buchwald et al., 2002; Schlumberger et al., 2003). SopE specifically targets the Rho GTPases Rac and Cdc42 in vitro whereas SopE2 only activates Cdc42 (Friebel et al., 2001). The role of Rac in Salmonella T3SS-1-dependent entry is well characterized but the role of Cdc42 is controversial. Criss et al. (2001) have demonstrated by using dominant negative forms and by pull-down assays that, in contrast to non-polarized cells, Cdc42 is not required and not activated during invasion of MCDK polarized epithelial cells. In the same way, Patel and Galan have observed that the depletion of Cdc42 by RNA interference (RNAi) in COS-2 and Henle-407 had no effect on membrane ruffling and efficient uptake (Patel \& Galan, 2006). In contrast, a recent genome-scale RNAi screening in HeLA cells indicated that Cdc42 depletion induced a $70 \%$ decrease in invasion, suggesting that it is required for the T3SS-1-dependent invasion process (Misselwitz et al., 2011). All together, it is difficult to conclude about the involvement of Cdc42 during T3SS-1-dependent entry.

Salmonella mutant strains lacking both sopE and sopE2 are still able to invade cells in a SopB dependent way (Zhou et al., 2001). SopB is an inositol phosphatase which shares homology with eukaryotic phopho-inositol (PI) phosphatases (Norris et al., 1998). Like SopE and SopE2, SopB targets a GTPase from the Rho family, RhoG, but in an indirect manner since SopB activates the GEF protein that allows RhoG activation (Patel \& Galan, 2006). Once 
activated, RhoG induces actin polymerization at the entry site presumably by stimulating the Arp2/3 complex (Patel \& Galan, 2006). A sopB deletion in S. Typhimurium induces a $50 \%$ decrease in invasion. But when sopB deletion is coupled with a sopE deletion, Salmonella uptake is drastically impaired (Zhou et al., 2001), demonstrating that all the T3SS-1 effectors work in concert to trigger entry.

Recently, SopB was also shown to manipulate actomyosin contractility to mediate invasion. SopB recruits myosin II by activating RhoA and its Rho kinase downstream effector. In contrast to the process leading to actin polymerization during Salmonella entry, myosin II recruitment at the entry site is independent on Arp2/3 nucleator activity (Hanisch et al., 2011).

In addition, different cellular proteins are involved during the T3SS-1 dependent entry of Salmonella without the identification of the bacterial effector responsible of this effect. This includes the focal adhesion kinase FAK, the Abelson tyrosine kinase Abl, and Shank3 (Shi \& Casanova, 2006; Huett et al., 2009; Ly \& Casanova, 2009). During Salmonella uptake, FAK acts as a scaffolding protein, but not as a protein tyrosine kinase and its interaction with p130Cas is involved in actin reorganization and membrane ruffle formation (Shi \& Casanova, 2006). But how Salmonella can nucleate assembly of focal adhesion-like complexes is still unclear and further research is needed to determine if this mechanism involves secreted bacterial effector proteins, other transmembrane host proteins, or both. Another scaffolding protein Shank3 also seems to regulate actin rearrangement during entry but the mechanisms leading to its recruitment and its activation have to be elucidated (Huett et al., 2009). As the experiments were performed using HeLa cells which are highly permissive to the T3SS-1dependent entry and with bacterial culture conditions that allow T3SS-1 expression, it is more probable that the Salmonella entry process inducing Shank3 recruitment is dependent on the T3SS-1. However, a T3SS-1-independent invasion process could also be involved because no mutant deficient for T3SS-1 expression was used as a control to analyze the involvement of Shank3.

Abelson tyrosine kinase $\mathrm{Abl}$ is also involved during Salmonella invasion as well as its effectors Abi1, a member of the WAVE2 complex, and CrkII (Ly \& Casanova, 2009). Abi1 could thus enhance actin polymerization at the entry site but the role of CrkII during the invasion process remains poorly characterized.

\subsubsection{Subversion of exocytosis machinery and membrane fusion during entry}

Recent data indicate that membrane fusion is a major process involved during entry, suggesting that membrane ruffling requires the addition of intracellular membrane. A study focusing on the subversion of the host exocyst complex during Salmonella entry has showed cooperation between SipC and SopE (Nichols \& Casanova, 2010). The exocyst is an octomeric complex (Sec3, Sec5, Sec6, Sec8, Sec10, Sec15, Exo70, Exo84) involved in vesicular trafficking which directs post-Golgi vesicules at specific site on the plasma membrane prior to their fusion. Nichols and Casanova have demonstrated that the mature exocyst complex is recruited at the entry site through an interaction between its subunit Exo70 and SipC. A depletion of exo70 or Sec5, another component of the exocyst complex, impairs S. Typhimurium invasion. Moreover, they have shown that SopE activates the Ras-related protein RalA, a small GTPase required for exocyst complex assembly (Nichols 
\& Casanova, 2010). It appears that SopE and SipC effectors manipulate the host exocyst to bring new membrane at the entry site in order to allow the formation of membrane ruffles and internalization.

SopB is also involved in this membrane fusion process through its inositol phosphatase activity. Dai et al. (2007) have shown that SopB-generated PI3P at the entry site leads to the recruitment of VAMP8, a host V-SNARE protein that mediates fusion between early and late endosomes. Moreover, depletion of VAMP8 by RNAi induces a decrease in invasion rate of a wild-type $S$. Typhimurium strain which is equivalent to that obtained with a sopB mutant. Thus, it seems that SopB promotes invasion by manipulating eukaryotic vesicular trafficking probably to induce fusion of intracellular vesicles to the cell membrane at the entry site.

How these events of vesicle-membrane fusion interact with actin cytoskeleton rearrangements to trigger entry has not been investigated yet. These different processes may synergize to induce internalization since actin dynamics is closely related to the metabolism of phosphoinosides (Honda et al., 1999; Sechi \& Wehland, 2000). However, VAMP8 which seems to be a marker of membrane fusion involved during $S$. Typhimurium invasion does not co-localize with F-actin during entry (Dai et al., 2007). In addition, it could be interesting to better characterize the role of the cellular factor IQGAP1 which is required for Salmonella uptake and acts following an interaction with actin and the RhoGTPases Rac1 and Cdc42 (Brown et al., 2007). Indeed, IQGAP1 is known to regulate actin architecture and interestingly, it also seems to act as a regulator of exocytosis by interacting with Exo70 (Rittmeyer et al., 2008). IQGAP1 could thus be one of the missing links between actin rearrangement and membrane fusion during Salmonella entry. Further studies could overcome this issue.

\subsubsection{Phagosome closure and restoration of the host cell normal shape}

As described above, inositol phosphatase activity of SopB drives to changes in cellular phosphoinosite concentrations at the bacteria/cell contact. In addition to generate PI3P at the entry site, translocation of SopB into host cells also induces $\mathrm{PI}(4,5) \mathrm{P}_{2}$ hydrolysis, which leads to an almost complete absence of $\mathrm{PI}(4,5) \mathrm{P}_{2}$ at the membrane invagination regions (Terebiznik et al., 2002). By reducing the local concentration of $\mathrm{PI}(4,5) \mathrm{P}_{2}$, SopB destabilizes cytoskeletonplasma membrane interactions, thus reducing the rigidity of the membrane and promoting invasion by facilitating the fission and the sealing of the future Salmonella-containing vacuole. In addition to SopB, SopD also contributes to membrane fission. Boonyom et al. have demonstrated that a $s o p D$ deletion, like the $\operatorname{sop} B$ mutant, leads to a decrease in membrane fission during invasion and that SopD is recruited at the bacterial invasion site dependently on the phosphatase activity of SopB (Bakowski et al., 2007). Thus, SopD seems to cooperate with SopB and contribute to Salmonella uptake by facilitating membrane fission at the entry site leading to the formation of the SCV (Bakowski et al., 2007).

Following the formation of intense membrane ruffling and internalization, the eukaryotic cell regains its normal shape, inducing the closure of the vacuole of endocytosis containing the bacteria. The restoration of actin cytoskeleton is promoted by the effector SptP, a tyrosine phosphatase which inactivates the RhoGTPases Rac- 1 and Cdc42 by stimulating their intrinsic GTPase activity (Fu \& Galan, 1998; Fu \& Galan, 1999). The N-terminal region of SptP interacts with Rac-1 and Cdc42 and a structural study of SptP indicates that this effector mimics the 
activity of host cell GAPs factors (Stebbins \& Galan, 2000). Interestingly, SptP is regulated by two different mechanisms in order to delay its activity in host cell compared to that of SopE or SipA. A microscopy analysis revealed that SipA is injected earlier than SptP in the host cytoplasm, which would imply that SipA has a higher affinity for the exportation apparatus of the T3SS-1 than SptP (Winnen et al., 2008). Moreover, SptP degradation by the host cell proteasome occurs later than SopE degradation (Kubori \& Galan, 2003).

\subsection{T3SS-1 contribution to Salmonella pathogenesis}

The T3SS-1 is the main invasion factor of Salmonella in vitro. Nevertheless, its contribution to pathogenesis depends on the model used. In vivo studies with $S$. Dublin and $S$. Typhimurium serotypes have demonstrated that the T3SS-1 is essential for intestinal colonization and is required to induce enterocolitis in bovine, rabbit and murine models (Wallis \& Galyov, 2000). In contrast, recent studies demonstrate that different serotypes of Salmonella lacking T3SS-1 still have the ability to invade in vitro cells of diverse origins and can be pathogenic in different in vivo infection models (Aiastui et al., 2010; Rosselin et al., 2011). In addition, it was shown that the T3SS-1 is not required for Salmonella internalization into a 3-Dimensional intestinal epithelium (Radtke et al., 2010). Moreover, a SPI-1 mutant of $S$. Gallinarum exhibits a reduced invasiveness into avian cells but is fully virulent in adult chicken (Jones et al., 2001). In S. Enteritidis and S. Typhimurium, the T3SS-1 is not essential during systemic infection of one week-old chicken or BalB/C mouse nor during the intestinal colonization of rabbit ileal loops (Coombes et al., 2005; Jones et al., 2007; Karasova et al., 2010). Moreover, S. Senftenberg strains lacking SPI-1 are isolated from human clinical cases, suggesting that the T3SS-1 is dispensable by this serotype for the establishment of infection in humans (Hu et al., 2008).

Taken together, these results indicate that T3SS-1- independent invasion mechanisms also play an important role in Salmonella infection and pathogenesis.

\section{Invasion mechanisms independent of the T3SS-1}

A Salmonella mutant, unable to express its T3SS-1 is still able to invade numerous cell lines and cell types and is shown to induce both intense and local membrane rearrangements (Rosselin et al., 2011). However, to date, little is known about the entry factors mediating these T3SS-1 independent invasion mechanisms. Here, we describe and sum up the state-of-art regarding these new invasion systems. Rck, PagN and HlyE are the three invasins identified as involved in Salmonella uptake. Moreover, Rosselin et al. (2011) have described that others unknown invasion factors exist although they are still not identified.

\subsection{The Rck invasin}

Among invasins that play a role in Salmonella invasion in a T3SS-1-independent way, Rck is clearly the best characterized. Rck is an $17 \mathrm{kDa}$ outer membrane protein (OMP) encoded by the large virulence plasmid of $S$. Enteritidis and $S$. Typhimurium (Heffernan et al., 1992; Rotger \& Casadesus, 1999). In addition to its ability to induce adhesion to and invasion of eukaryotic cells, Rck confers a high resistance level to complement killing by preventing the formation of the membrane attack complex (Heffernan et al., 1992; Cirillo et al., 1996; Rosselin et al., 2010). 


\subsubsection{Rck structure}

Rck is a member of an outer membrane protein family named "Ail/Lom family". This family consists of five members (Rck, Ail, Lom, OmpX and PagC) which are predicted to have eight transmembrane beta-sheets and four cell surface-exposed loops. Even if these proteins present a similar conformation, they have different functions. Rck and Ail (encoded by a chromosomal gene of Yersinia Enterocolitica) share the ability to promote serum resistance and epithelial cell invasion. These proteins do not exhibit homologous regions that could be related to these two identical roles. In Ail, the cell invasion property is associated with loop2 whereas loop3 and more precisely the region from the amino acids G113 to V159 was shown to be the minimal region of Rck required and sufficient for cell adhesion and invasion (Miller et al., 2001; Rosselin et al., 2010).

Another member of this family involved in virulence is PagC which is encoded by a phoPregulated gene on the Salmonella chromosome and plays a role in intracellular macrophage survival in Salmonella (Miller et al., 1989; Gunn et al., 1995). Others members of this family are OmpX of Enterobacter cloacae (Mecsas et al., 1995) and Lom, a protein expressed by bacteriophage $\lambda$ from lysogenic $E$. coli (Reeve \& Shaw, 1979) but none of them have known virulence-associated phenotype.

\subsubsection{Rck regulation}

A genetic screening performed in $S$. Typhimurium to identify genes regulated by SdiA (suppressor of division inhibition), a transcriptional regulator of quorum sensing, has suggested that $r c k$ belongs to a putative operon called the "rck operon" whose expression is activated by SdiA in an Acyl Homoserine Lactone (AHLs)-dependent manner (Figure 6) (Ahmer et al., 1998; Michael et al., 2001).

The $r c k$ operon contains 6 open reading frames: $p e f I, \operatorname{srg} D, \operatorname{srg} A, \operatorname{srg} B, r c k$ and $\operatorname{srg} C$ (Figure 6). Two genes in this operon, pefI (plasmid encoded fimbriae) and $\operatorname{srg} A$ (sdiA-regulated gene), affect the expression and function of the pef operon located upstream of the rck operon and involved in the biosynthesis of the Pef fimbriae. pefI encodes a transcriptional regulator of the pef operon, and SrgA is a DsbA paralog that efficiently oxidizes the disulfide bond of PefA, the major structural subunit of the Pef fimbriae (Bouwman et al., 2003). These fimbriae are involved in biofilm formation, adhesion to murine small intestine and fluid accumulation in the infant mouse (Baumler et al., 1996; Ledeboer et al., 2006). Also localized on the $r c k$ operon, $s r g D$ encodes a putative transcriptional regulator. Recently, it has been shown that SrgD acts in cooperation with PefI to induce a synergistic negative regulation of flagellar genes expression (Wozniak et al., 2009; Wallar et al., 2011). The remaining genes on the $r c k$ operon have unknown functions and encode a putative outer membrane protein, $\mathrm{SrgB}$, and a putative transcriptional regulator, $\mathrm{SrgC}$.

Another locus regulated by SdiA-AHLs has been identified during screening. This chromosomal locus encodes a single gene named srgE (STM1554) (Ahmer et al., 1998). No function for SrgE is described but a computational approach has suggested that SrgE is secreted by the T3SS-1 (Samudrala et al., 2009) (Figure 6).

As E. coli and Klebsiella, Salmonella lack an AHL synthase and thus do not produce AHLs. However, SdiA can detect and bind AHLs produced by others bacterial species (Michael et al., 2001). SdiA is a LuxR homologue and has two functional domains. The C-terminal 
region contains a predicted helix-turn-helix motif implicated in DNA binding and a Nterminal domain called "autoinducer domain" that interacts with AHLs. By NMR analysis, Yao et al. (2007) have shown that a direct interaction between SdiA and AHLs is required for SdiA folding and function.

Temperature also affects rck operon expression. At temperature below $30^{\circ} \mathrm{C}$, the transcription of $r c k$ operon is repressed, while $\operatorname{srg} E$ is repressed only at temperature below $22^{\circ} \mathrm{C}$ (Smith \& Ahmer, 2003)(Figure 6). As SdiA expression is not temperature regulated, another level of $r c k$ operon regulation remains to be identified.

In addition, a transcriptomic study has shown that Hha and its paralogue $\mathrm{YdgH}$ could be involved in the regulation of the $r c k$ operon (Vivero et al., 2008).

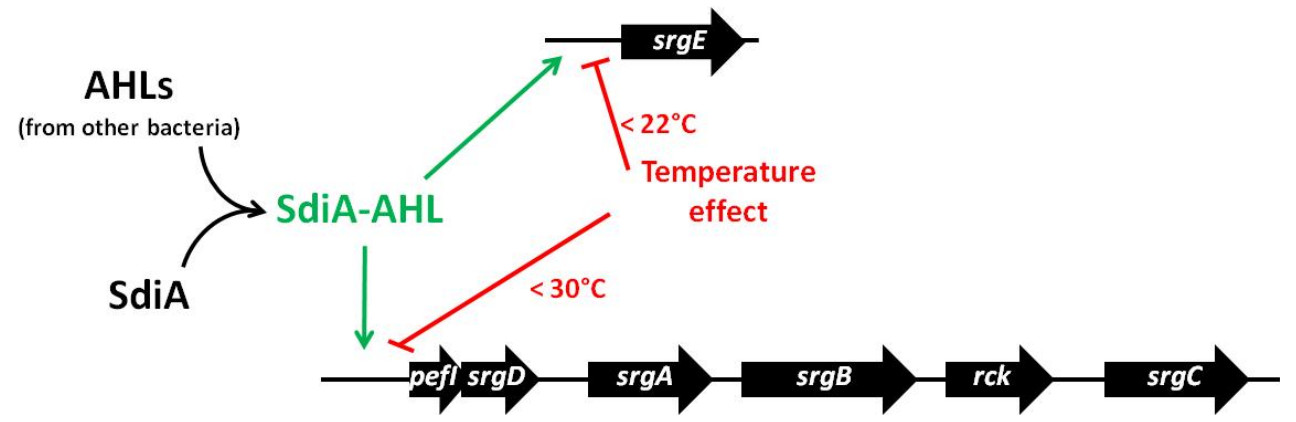

Fig. 6. Regulation of $r c k$ operon expression. When bound to AHLs, SdiA activates the expression of $r c k$ operon and $s r g E$. Under temperatures that are lower than $30^{\circ} \mathrm{C}$ or $22^{\circ} \mathrm{C}$, the expression of $r c k$ operon and $s r g E$ is inhibited, respectively.

\subsubsection{Rck-mediated entry mechanism}

When a $r c k$ mutant is grown under swarming conditions known to induce SdiA expression, a 2-3 fold decrease in epithelial cell invasion has been observed compared to the wild-type strain (Rosselin et al., 2010). Moreover, in standard culture conditions, Rck overexpression leads to an increase in invasion.

By using both an initially non-invasive E. coli strain overexpressing Rck and latex beads coated with the minimal region of Rck inducing invasion (G113-V159), it was demonstrated that Rck alone is able to induce entry by a receptor-mediated process. This mechanism promotes local actin remodelling and weak and closely adherent membrane extensions (Rosselin et al., 2010). Salmonella can thus enter cells through two distinct mechanisms: the Trigger mechanism mediated by its T3SS-1 apparatus and a Zipper mechanism induced by Rck. A model of this Zipper entry process is shown in figure 7. Following an interaction between Rck and its unknown cellular receptor, it was shown by using specific drugs and a dominant negative form that the class I PI3-kinase made of the p85-p110 heterodimer is required for Rck mediated entry. Moreover, Rck induces an increase in the interactions between p85 and phosphotyrosine residues, leading to the class I PI3-Kinase activation. Pharmacological approaches or Akt knockout cells also demonstrate that Akt is necessary to Rck-mediated internalization. Probably by binding to $\mathrm{PI}(3,4,5) \mathrm{P}_{3}$, Akt is recruited at the entry site and activated in a PI3-Kinase dependent way (Mijouin et al., 2012). 
The GTPase Rho is not involved during the Rck entry process but the use of dominant negatives demonstrates that Rac1 and Cdc42 are required (Rosselin et al., 2010). Moreover, Rac1 is recruited at the entry site and Rck induces an increase in the level of active Rac1, demonstrating that it activates this GTPase (Rosselin et al., 2010; Mijouin et al., 2012). Rac1 activation occurs downstream on the PI-3kinase activity. Finally, overexpression of inhibitory constructs has shown that actin polymerization is dependent on the Arp2/3 nucleator complex during Rck-mediated entry (Rosselin et al., 2010).

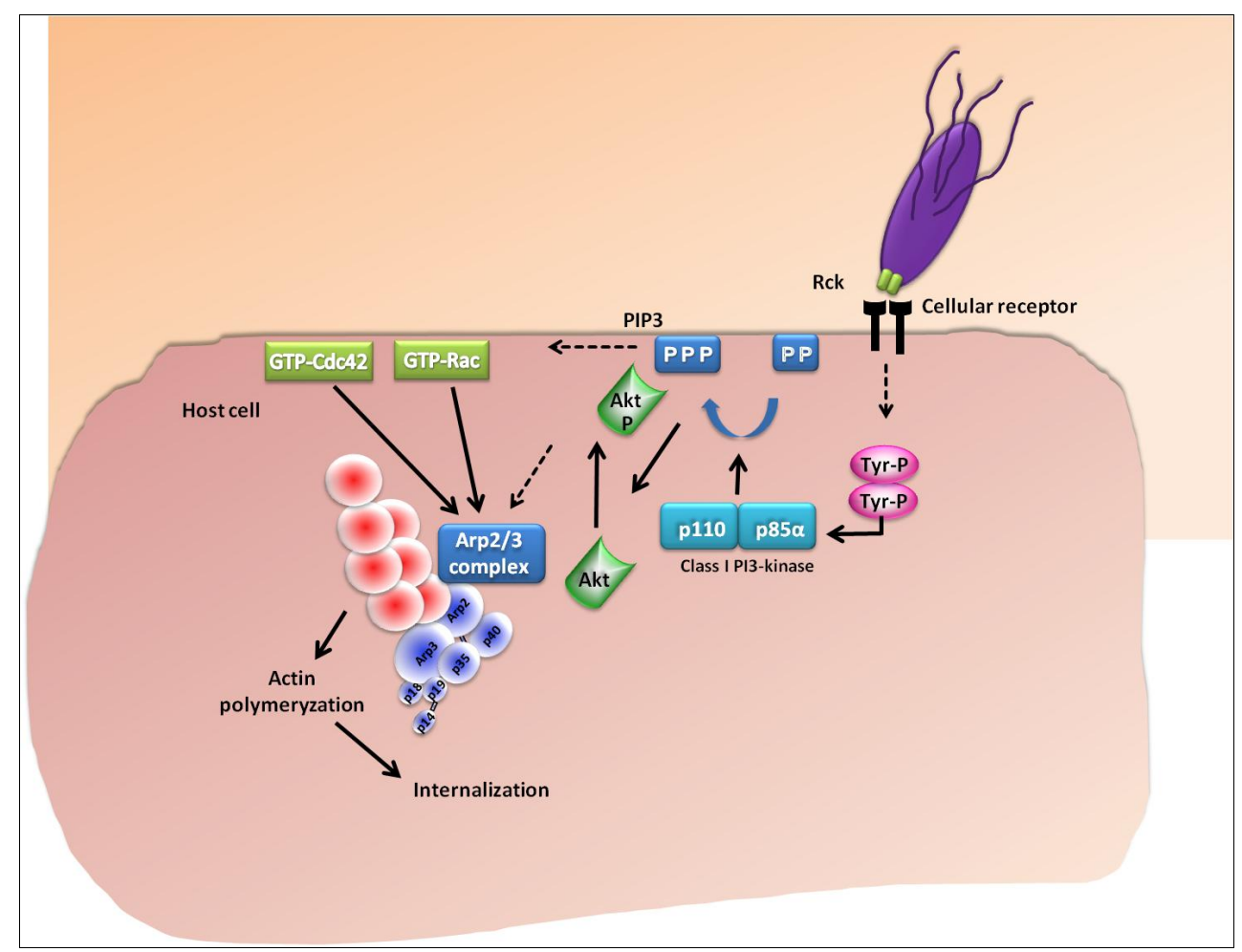

Fig. 7. Model of the cellular signaling pathway induces by Rck and leading to invasion. This Zipper entry process involves different cellular proteins: Class I PI3-Kinase, Akt, Rac and Cdc42, the Arp2/3 complex and actin. Dotted arrows: possible signalling events and/or interactions.

\subsubsection{Rck contribution to Salmonella pathogenesis}

The role of Rck in Salmonella invasion is clearly demonstrated in vitro, but its role in Salmonella pathogenesis is poorly understood. Indeed, in vivo conditions allowing rck expression are unclear. The fact that Rck is regulated by SdiA, a quorum sensing regulator suggests an intestinal role of this invasin (Ahmer et al., 1998). However, the mechanisms leading to SdiA activation and $r c k$ expression are not well characterized. SdiA activation has been investigated in different hosts including rabbit, guinea pig, cow, turtle, mouse, pig and chicken but no activated-SdiA has been detected in the gastro-intestinal tract of 
these animals, except for turtles which were found to be co-infected with AHLsproducing Aeromonas hydrophila (Smith et al., 2008). Another work has demonstrated that SdiA is activated in mice previously infected with Yersinia enterocolitica, which is able to synthesize AHLs (Dyszel et al., 2010). However, in these conditions, SdiA activation does not confer a fitness advantage for Salmonella intestinal colonization in comparison to a sdiA mutant (Dyszel et al., 2010). These results suggest that even if SdiA activation is achieved when AHL-producing strains colonize the gastrointestinal tract, it is not always sufficient to induce the expression of its regulon including $r c k$. To assess the role of SdiA and its regulon during intestinal infection, Dyszel et al. (2010) have constructed a Salmonella strain able to produce AHLs. After co-infection of mice with the AHLproducing Salmonella strain and a sdiA mutant, it was shown that the constant activation of SdiA confers a selective advantage to Salmonella. Moreover, a loss of this selective advantage was observed when all individual SdiA-regulated genes were deleted, including $r c k$, suggesting a role during intestinal colonization. Nevertheless, an in vivo model allowing a physiological activation of SdiA would be needed to assess the contribution of Rck to intestinal infection.

In addition, as $r c k$ is also regulated by an unidentified SdiA-independent system (Smith et al., 2008), it is conceivable that Rck invasion mechanism is not restricted to the gastrointestinal tract. Considering that Rck is also involved in the resistance to complement killing, a systemic function of Rck is possible.

\subsection{The PagN invasin}

In addition to the T3SS-1 and Rck, the PagN outer membrane protein has also been identified as being involved in Salmonella invasion (Lambert \& Smith, 2008). pagN, is localized on the centisome 7 genomic island and is widely distributed among Salmonella enterica serotypes (Folkesson et al., 1999). The pagN ORF was originally identified during a TnphoA random-insertion screening in $S$. Typhimurium performed to discover PhoPactivated genes (Belden \& Miller, 1994).

\subsubsection{PagN structure}

PagN shares similarity to both the Tia and Hek invasins of E. coli, and presents $39 \%$ and $42 \%$ identity in amino acids with these two invasins, respectively. Tia and Hek are predicted to have eight transmembrane regions, four long exposed extracellular loops and three short periplasmic turns (Mammarappallil \& Elsinghorst, 2000; Fagan et al., 2008). Thus, PagN probably adopts a similar conformation as that of Hek and Tia.

\subsubsection{PagN regulation}

The pagN (phoP-activated gene) gene is phoP-regulated. The PhoP/PhoQ system is a twocomponent transcriptional regulatory system which modulates transcription of a multitude of virulence genes in Salmonella. This regulatory system is composed of the PhoQ sensor kinase (located at the membrane) and the PhoP response regulator. In response to specific stimuli such as acidified macrophage phagosome environment or low $\mathrm{Mg}^{2+}$ concentration, PhoQ is auto-phosphorylated and transfers its phosphates to the cytoplasmic DNA-binding protein PhoP, regulating specific genes. 


\subsubsection{PagN-mediated entry mechanism}

Lambert \& Smith (2008) have demonstrated that pagN deletion in S. Typhimurium leads to a 3 -fold decrease in invasion of enterocytes without altering cell adhesion. At the cellular level, the PagN-mediated entry process is poorly characterized. It was only shown that actin polymerization is required during invasion (Lambert \& Smith, 2008) and that PagN is able to interact with extracellular heparin proteoglycans (Lambert \& Smith, 2009). However, proteoglycans cannot transduce a signaling cascade so it is probable that they act as co-receptors for invasion and not as the receptor itself. Moreover, although an interaction between PagN and heparin has been suggested, no clear heparin-binding motif was detected. Moreover, all PagN loops are required for invasion in epithelial cells (Lambert \& Smith, 2009).

\subsubsection{PagN contribution to Salmonella pathogenesis}

PagN is required for survival in BALB/c mice (Heithoff et al., 1999) and a pagN mutant is less competitive to colonize the spleen of mice compared to its parental strain (Conner et al., 1998). However, the role of PagN in Salmonella pathogenesis is still unclear. pagN is activated by PhoP and thus maximally expressed intracellularly, a condition in which the SPI-1 island encoding the T3SS-1 is downregulated (Conner et al., 1998; Heithoff et al., 1999; Eriksson et al., 2003). Lambert \& Smith (2008) thus postulate that bacteria which exit epithelial cells or macrophages have an optimal level of PagN expression, but have a low T3SS-1 expression and this might facilitate subsequent interactions with mammalian cells that the pathogen encounters after host cell destruction.

\subsection{The HlyE invasin}

The hlyE gene is localized on the Salmonella pathogenicity island SPI-18 and is expressed by serotypes associated with systemic infection in humans including $S$. Typhi and $S$. Paratyphi A (Fuentes et al., 2008). The hlyE sequence shares more than $90 \%$ identity with that of Escherichia coli HlyE (ClyA) hemolysin. The HlyE protein is able to lyse epithelial cells when exported from bacterial cell via outer membrane vesicule release (Wai et al., 2003). Recently, Fuentes et al. (2008) have demonstratred that HlyE contributes to epithelial cell invasion of $S$. Typhi. However, the cellular events leading to HlyE-mediated invasion have not been characterized.

In vivo studies have shown that HlyE contributes to colonization of mouse deep organs (Fuentes et al., 2008). However, how HlyE participates in establishment of systemic infection of Salmonella is not well understood.

\subsection{Non-identified invasion factors}

Several studies have revealed that invasion systems in $S$. Typhimurium and $S$. Enteritidis are not restricted to the T3SS-1, Rck and PagN. Indeed, a strain which does not express rck, pagN and the T3SS- 1 is still able to significantly invade fibroblasts, epithelial and endothelial cells (Rosselin et al., 2011). Results obtained by Aiastui et al. (2010) and van Sorge et al. (2011) have reinforced the idea that non-identified invasion factors are involved during entry into these cell types since Salmonella strains that cannot express the 
T3SS-1 still enter into epithelial cells, endothelial cells and fibroblasts in a significant way. Moreover, invasion analyses of a 3-D intestinal epithelium by $S$. Typhimurium have also highlighted the fact that Salmonella possess non-characterized invasion factors (Radtke et al., 2010).

Actin network remodeling and membrane rearrangements induced by these unknown factors have been visualized by confocal and electron microscopy as well as both Zipperlike and Trigger-like membrane alterations (Rosselin et al., 2011). Identification of these factors is required to confirm these observations. It suggests that Salmonella express nonidentified invasins able to mediate a Zipper process and factor(s) other than the T3SS-1 that induce Trigger-like invasion process(es). Type IV or type VI secretion systems are good candidates to induce Trigger-like cellular structures as they are able to translocate proteins directly into the host cell cytosol and as they are major virulence determinants involved in the pathogenesis of diverse Gram-negative bacteria (Oliveira et al., 2006; Filloux et al., 2008; Blondel et al., 2010).

These observations thus open new avenues for identification of new invasion factors.

\section{Conclusions and perspectives}

Until recently, it was accepted that Salmonella enter cells only via its T3SS-1, which mediates a Trigger entry process. The T3SS-1-dependent invasion system has been widely described in the literature both at the bacterial and the cellular molecular levels. Moreover, the requirement of the T3SS-1 during intestinal and systemic infections has been demonstrated in some animals (Wallis \& Galyov, 2000). However, an increasing number of reports describe that different serotypes of Salmonella can induce host infection without a functional T3SS-1 (Penheiter et al., 1997; Jones et al., 2001; Karasova et al., 2010). This has been demonstrated with T3SS-1 mutants but also with clinical Salmonella strains (Hu et al., 2008). However, the majority of Salmonella invasion system studies have focused on the T3SS-1 and we have little information concerning the T3SS-1-independent entry processes. Several invasins including PagN, Rck and HlyE have been recently identified in Salmonella and different investigations have provided evidences for other non-identified invasion factors (Aiastui et al., 2010; Radtke et al., 2010; Rosselin et al., 2011; van Sorge et al., 2011). In addition, the vast majority of this information has been obtained in a mouse model and with $S$. Typhimurium and much less data are available for other serotypes especially those adapted to pigs, cattle or poultry which represent major reservoirs of Salmonella. Salmonella enterica contain, over 2,500 diverse serotypes that have different host ranges, and cause diseases with severity ranging from subclinical colonization to serious systemic disease. Because the essential feature of the pathogenicity of Salmonella is its interaction with host cells, the identification of new entry routes could, in part, explain their different host ranges and disease symptoms.

The finding that Salmonella serotypes use different cell receptors and cell routes for host infection shows that the contribution of Salmonella genes to pathogenesis may be more complex than previously thought. These findings are changing our classical view of Salmonella pathogenicity. This new paradigm will modify the understanding of the mechanisms that lead to the different Salmonella-induced diseases and could allow us to revisit the host specificity bases. 


\section{References}

Ahmer, B. M., van Reeuwijk, J., Timmers, C. D., Valentine, P. J. \& Heffron, F. (1998). Salmonella typhimurium encodes an SdiA homolog, a putative quorum sensor of the LuxR family, that regulates genes on the virulence plasmid. J Bacteriol. Vol. 180, No. 5, pp. 1185-93.

Aiastui, A., Pucciarelli, M. G. \& Garcia-del Portillo, F. (2010). Salmonella enterica serovar typhimurium invades fibroblasts by multiple routes differing from the entry into epithelial cells. Infect Immun. Vol. 78, No. 6, pp. 2700-13.

Akbar, S., Schechter, L. M., Lostroh, C. P. \& Lee, C. A. (2003). AraC/XylS family members, HilD and HilC, directly activate virulence gene expression independently of HilA in Salmonella typhimurium. Mol Microbiol. Vol. 47, No. 3, pp. 715-28.

Akeda, Y. \& Galan, J. E. (2004). Genetic analysis of the Salmonella enterica type III secretionassociated ATPase InvC defines discrete functional domains. J Bacteriol. Vol. 186, No. 8, pp. 2402-12.

Bakowski, M. A., Cirulis, J. T., Brown, N. F., Finlay, B. B. \& Brumell, J. H. (2007). SopD acts cooperatively with SopB during Salmonella enterica serovar Typhimurium invasion. Cell Microbiol. Vol. 9, No. 12, pp. 2839-55.

Baumler, A. J., Tsolis, R. M., Bowe, F. A., Kusters, J. G., Hoffmann, S. \& Heffron, F. (1996). The pef fimbrial operon of Salmonella typhimurium mediates adhesion to murine small intestine and is necessary for fluid accumulation in the infant mouse. Infect Immun. Vol. 64, No. 1, pp. 61-8.

Baxter, M. A., Fahlen, T. F., Wilson, R. L. \& Jones, B. D. (2003). HilE interacts with HilD and negatively regulates hilA transcription and expression of the Salmonella enterica serovar Typhimurium invasive phenotype. Infect Immun. Vol. 71, No. 3, pp. 1295-305.

Baxter, M. A. \& Jones, B. D. (2005). The fimYZ genes regulate Salmonella enterica Serovar Typhimurium invasion in addition to type 1 fimbrial expression and bacterial motility. Infect Immun. Vol. 73, No. 3, pp. 1377-85.

Belden, W. J. \& Miller, S. I. (1994). Further characterization of the PhoP regulon: identification of new PhoP-activated virulence loci. Infect Immun. Vol. 62, No. 11, pp. 5095-101.

Blondel, C. J., Yang, H. J., Castro, B., Chiang, S., Toro, C. S., Zaldivar, M., Contreras, I., Andrews-Polymenis, H. L. \& Santiviago, C. A. (2010). Contribution of the type VI secretion system encoded in SPI-19 to chicken colonization by Salmonella enterica serotypes Gallinarum and Enteritidis. PLoS One. Vol. 5, No. 7, pp. e11724.

Boonyom, R., Karavolos, M.H., Bulmer, D.M. \& Khan, C.M. (2010). /Salmonellal pathogenicity island 1 (SPI-1) type III secretion of SopD involves N- and C-terminal signals and direct binding to the InvC ATPase. Microbiology. vol.156, No.6, pp 1805-14.

Bouwman, C. W., Kohli, M., Killoran, A., Touchie, G. A., Kadner, R. J. \& Martin, N. L. (2003). Characterization of SrgA, a Salmonella enterica serovar Typhimurium virulence plasmid-encoded paralogue of the disulfide oxidoreductase DsbA, essential for biogenesis of plasmid-encoded fimbriae. J Bacteriol. Vol. 185, No. 3, pp. 991-1000.

Bretscher, M. S. (1991). Lipid flow in locomoting cells. Science. Vol. 251, No. 4991, pp. 317-8.

Brown, M. D., Bry, L., Li, Z. \& Sacks, D. B. (2007). IQGAP1 regulates Salmonella invasion through interactions with actin, Rac1, and Cdc42. J Biol Chem. Vol. 282, No. 41, pp. 30265-72. 
Buchwald, G., Friebel, A., Galan, J. E., Hardt, W. D., Wittinghofer, A. \& Scheffzek, K. (2002). Structural basis for the reversible activation of a Rho protein by the bacterial toxin SopE. EMBO J. Vol. 21, No. 13, pp. 3286-95.

Chang, J., Chen, J. \& Zhou, D. (2005). Delineation and characterization of the actin nucleation and effector translocation activities of Salmonella SipC. Mol Microbiol. Vol. 55, No. 5, pp. 1379-89.

Chang, J., Myeni, S. K., Lin, T. L., Wu, C. C., Staiger, C. J. \& Zhou, D. (2007). SipC multimerization promotes actin nucleation and contributes to Salmonella-induced inflammation. Mol Microbiol. Vol. 66, No. 6, pp. 1548-56.

Cirillo, D. M., Heffernan, E. J., Wu, L., Harwood, J., Fierer, J. \& Guiney, D. G. (1996). Identification of a domain in Rck, a product of the Salmonella typhimurium virulence plasmid, required for both serum resistance and cell invasion. Infect Immun. Vol. 64, No. 6, pp. 2019-23.

Conner, C. P., Heithoff, D. M. \& Mahan, M. J. (1998). In vivo gene expression: contributions to infection, virulence, and pathogenesis. Curr Top Microbiol Immunol. Vol. 225, No., pp. 1-12.

Coombes, B. K., Wickham, M. E., Lowden, M. J., Brown, N. F. \& Finlay, B. B. (2005). Negative regulation of Salmonella pathogenicity island 2 is required for contextual control of virulence during typhoid. Proc Natl Acad Sci U S A. Vol. 102, No. 48, pp. 17460-5.

Cossart, P. \& Sansonetti, P. J. (2004). Bacterial invasion: the paradigms of enteroinvasive pathogens. Science. Vol. 304, No. 5668, pp. 242-8.

Criss, A. K., Ahlgren, D. M., Jou, T. S., McCormick, B. A. \& Casanova, J. E. (2001). The GTPase Rac1 selectively regulates Salmonella invasion at the apical plasma membrane of polarized epithelial cells. J Cell Sci. Vol. 114, No. Pt 7, pp. 1331-41.

Dai, S., Zhang, Y., Weimbs, T., Yaffe, M. B. \& Zhou, D. (2007). Bacteria-generated PtdIns(3)P recruits VAMP8 to facilitate phagocytosis. Traffic. Vol. 8, No. 10, pp. 1365-74.

Darwin, K. H. \& Miller, V. L. (1999). InvF is required for expression of genes encoding proteins secreted by the SPI type III secretion apparatus in Salmonella typhimurium. J Bacteriol. Vol. 181, No. 16, pp. 4949-54.

Dyszel, J. L., Smith, J. N., Lucas, D. E., Soares, J. A., Swearingen, M. C., Vross, M. A., Young, G. M. \& Ahmer, B. M. (2010). Salmonella enterica serovar Typhimurium can detect acyl homoserine lactone production by Yersinia enterocolitica in mice. J Bacteriol. Vol. 192, No. 1, pp. 29-37.

Ellermeier, C. D. \& Slauch, J. M. (2004). RtsA coordinately regulates DsbA and the Salmonella pathogenicity island 1 type III secretion system. J Bacteriol. Vol. 186, No. 1, pp. 68-79.

Ellermeier, J. R. \& Slauch, J. M. (2007). Adaptation to the host environment: regulation of the SPI1 type III secretion system in Salmonella enterica serovar Typhimurium. Curr Opin Microbiol. Vol. 10, No. 1, pp. 24-9.

Eriksson, S., Lucchini, S., Thompson, A., Rhen, M. \& Hinton, J. C. (2003). Unravelling the biology of macrophage infection by gene expression profiling of intracellular Salmonella enterica. Mol Microbiol. Vol. 47, No. 1, pp. 103-18.

Fagan, R. P., Lambert, M. A. \& Smith, S. G. (2008). The hek outer membrane protein of Escherichia coli strain RS218 binds to proteoglycan and utilizes a single extracellular loop for adherence, invasion, and autoaggregation. Infect Immun. Vol. 76, No. 3, pp. 1135-42.

Fahlen, T. F., Mathur, N. \& Jones, B. D. (2000). Identification and characterization of mutants with increased expression of hilA, the invasion gene transcriptional activator of Salmonella typhimurium. FEMS Immunol Med Microbiol. Vol. 28, No. 1, pp. 25-35. 
Filloux, A., Hachani, A. \& Bleves, S. (2008). The bacterial type VI secretion machine: yet another player for protein transport across membranes. Microbiology. Vol. 154, No. Pt 6, pp. 1570-83.

Folkesson, A., Advani, A., Sukupolvi, S., Pfeifer, J. D., Normark, S. \& Lofdahl, S. (1999). Multiple insertions of fimbrial operons correlate with the evolution of Salmonella serovars responsible for human disease. Mol Microbiol. Vol. 33, No. 3, pp. 612-22.

Friebel, A., Ilchmann, H., Aepfelbacher, M., Ehrbar, K., Machleidt, W. \& Hardt, W. D. (2001). SopE and SopE2 from Salmonella typhimurium activate different sets of RhoGTPases of the host cell. J Biol Chem. Vol. 276, No. 36, pp. 34035-40.

Fu, Y. \& Galan, J. E. (1998). The Salmonella typhimurium tyrosine phosphatase SptP is translocated into host cells and disrupts the actin cytoskeleton. Mol Microbiol. Vol. 27, No. 2, pp. 359-68.

Fu, Y. \& Galan, J. E. (1999). A Salmonella protein antagonizes Rac-1 and Cdc42 to mediate host-cell recovery after bacterial invasion. Nature. Vol. 401, No. 6750, pp. 293-7.

Fuentes, J. A., Villagra, N., Castillo-Ruiz, M. \& Mora, G. C. (2008). The Salmonella Typhi hlyE gene plays a role in invasion of cultured epithelial cells and its functional transfer to S. Typhimurium promotes deep organ infection in mice. Res Microbiol. Vol. 159, No. 4, pp. 279-87.

Galan, J. E. \& Wolf-Watz, H. (2006). Protein delivery into eukaryotic cells by type III secretion machines. Nature. Vol. 444, No. 7119, pp. 567-73.

Galkin, V. E., Orlova, A., VanLoock, M. S., Zhou, D., Galan, J. E. \& Egelman, E. H. (2002). The bacterial protein SipA polymerizes G-actin and mimics muscle nebulin. Nat Struct Biol. Vol. 9, No. 7, pp. 518-21.

Garner, M. J., Hayward, R. D. \& Koronakis, V. (2002). The Salmonella pathogenicity island 1 secretion system directs cellular cholesterol redistribution during mammalian cell entry and intracellular trafficking. Cell Microbiol. Vol. 4, No. 3, pp. 153-65.

Groisman, E. A. \& Ochman, H. (1993). Cognate gene clusters govern invasion of host epithelial cells by Salmonella typhimurium and Shigella flexneri. EMBO J. Vol. 12, No. 10, pp. 3779-87.

Gunn, J. S., Alpuche-Aranda, C. M., Loomis, W. P., Belden, W. J. \& Miller, S. I. (1995). Characterization of the Salmonella typhimurium pagC/pagD chromosomal region. J Bacteriol. Vol. 177, No. 17, pp. 5040-7.

Hanisch, J., Kolm, R., Wozniczka, M., Bumann, D., Rottner, K. \& Stradal, T. E. (2011). Activation of a RhoA/myosin II-dependent but Arp2/3 complex-independent pathway facilitates Salmonella invasion. Cell Host Microbe. Vol. 9, No. 4, pp. 273-85.

Hartwig, J. H. \& Kwiatkowski, D. J. (1991). Actin-binding proteins. Curr Opin Cell Biol. Vol. 3, No. 1, pp. 87-97.

Hayward, R. D., Cain, R. J., McGhie, E. J., Phillips, N., Garner, M. J. \& Koronakis, V. (2005). Cholesterol binding by the bacterial type III translocon is essential for virulence effector delivery into mammalian cells. Mol Microbiol. Vol. 56, No. 3, pp. 590-603.

Hayward, R. D., McGhie, E. J. \& Koronakis, V. (2000). Membrane fusion activity of purified SipB, a Salmonella surface protein essential for mammalian cell invasion. Mol Microbiol. Vol. 37, No. 4, pp. 727-39.

Heffernan, E. J., Harwood, J., Fierer, J. \& Guiney, D. (1992). The Salmonella typhimurium virulence plasmid complement resistance gene rck is homologous to a family of virulence-related outer membrane protein genes, including pagC and ail. J Bacteriol. Vol. 174, No. 1, pp. 84-91. 
Heithoff, D. M., Conner, C. P., Hentschel, U., Govantes, F., Hanna, P. C. \& Mahan, M. J. (1999). Coordinate intracellular expression of Salmonella genes induced during infection. J Bacteriol. Vol. 181, No. 3, pp. 799-807.

Higashide, W., Dai, S., Hombs, V. P. \& Zhou, D. (2002). Involvement of SipA in modulating actin dynamics during Salmonella invasion into cultured epithelial cells. Cell Microbiol. Vol. 4, No. 6, pp. 357-65.

Honda, A., Nogami, M., Yokozeki, T., Yamazaki, M., Nakamura, H., Watanabe, H., Kawamoto, K., Nakayama, K., Morris, A. J., Frohman, M. A. \& Kanaho, Y. (1999). Phosphatidylinositol 4-phosphate 5-kinase alpha is a downstream effector of the small G protein ARF6 in membrane ruffle formation. Cell. Vol. 99, No. 5, pp. 521-32.

Hu, Q., Coburn, B., Deng, W., Li, Y., Shi, X., Lan, Q., Wang, B., Coombes, B. K. \& Finlay, B. B. (2008). Salmonella enterica serovar Senftenberg human clinical isolates lacking SPI-1. J Clin Microbiol. Vol. 46, No. 4, pp. 1330-6.

Hueck, C. J. (1998). Type III protein secretion systems in bacterial pathogens of animals and plants. Microbiol Mol Biol Rev. Vol. 62, No. 2, pp. 379-433.

Hueck, C. J., Hantman, M. J., Bajaj, V., Johnston, C., Lee, C. A. \& Miller, S. I. (1995). Salmonella typhimurium secreted invasion determinants are homologous to Shigella Ipa proteins. Mol Microbiol. Vol. 18, No. 3, pp. 479-90.

Huett, A., Leong, J. M., Podolsky, D. K. \& Xavier, R. J. (2009). The cytoskeletal scaffold Shank3 is recruited to pathogen-induced actin rearrangements. Exp Cell Res. Vol. 315, No. 12, pp. 2001-11.

Ibarra, J. A. \& Steele-Mortimer, O. (2009). Salmonella--the ultimate insider. Salmonella virulence factors that modulate intracellular survival. Cell Microbiol. Vol. 11, No. 11, pp. 1579-86.

Jones, M. A., Hulme, S. D., Barrow, P. A. \& Wigley, P. (2007). The Salmonella pathogenicity island 1 and Salmonella pathogenicity island 2 type III secretion systems play a major role in pathogenesis of systemic disease and gastrointestinal tract colonization of Salmonella enterica serovar Typhimurium in the chicken. Avian Pathol. Vol. 36, No. 3, pp. 199-203.

Jones, M. A., Wigley, P., Page, K. L., Hulme, S. D. \& Barrow, P. A. (2001). Salmonella enterica serovar Gallinarum requires the Salmonella pathogenicity island 2 type III secretion system but not the Salmonella pathogenicity island 1 type III secretion system for virulence in chickens. Infect Immun. Vol. 69, No. 9, pp. 5471-6.

Kaniga, K., Trollinger, D. \& Galan, J. E. (1995). Identification of two targets of the type III protein secretion system encoded by the inv and spa loci of Salmonella typhimurium that have homology to the Shigella IpaD and IpaA proteins. J Bacteriol. Vol. 177, No. 24, pp. 7078-85.

Karasova, D., Sebkova, A., Havlickova, H., Sisak, F., Volf, J., Faldyna, M., Ondrackova, P., Kummer, V. \& Rychlik, I. (2010). Influence of 5 major Salmonella pathogenicity islands on NK cell depletion in mice infected with Salmonella enterica serovar Enteritidis. BMC Microbiol. Vol. 10, No., pp. 75.

Kimbrough, T. G. \& Miller, S. I. (2000). Contribution of Salmonella typhimurium type III secretion components to needle complex formation. Proc Natl Acad Sci U S A. Vol. 97, No. 20, pp. 11008-13.

Kimbrough, T. G. \& Miller, S. I. (2002). Assembly of the type III secretion needle complex of Salmonella typhimurium. Microbes Infect. Vol. 4, No. 1, pp. 75-82.

Kubori, T. \& Galan, J. E. (2003). Temporal regulation of Salmonella virulence effector function by proteasome-dependent protein degradation. Cell. Vol. 115, No. 3, pp. 333-42. 
Lambert, M. A. \& Smith, S. G. (2008). The PagN protein of Salmonella enterica serovar Typhimurium is an adhesin and invasin. BMC Microbiol. Vol. 8, No., pp. 142.

Lambert, M. A. \& Smith, S. G. (2009). The PagN protein mediates invasion via interaction with proteoglycan. FEMS Microbiol Lett. Vol. 297, No. 2, pp. 209-16.

Lara-Tejero, M., Kato, J., Wagner, S., Liu, X. \& Galan, J. E. (2011). A sorting platform determines the order of protein secretion in bacterial type III systems. Science. Vol. 331, No. 6021, pp. 1188-91.

Ledeboer, N. A., Frye, J. G., McClelland, M. \& Jones, B. D. (2006). Salmonella enterica serovar Typhimurium requires the Lpf, Pef, and Tafi fimbriae for biofilm formation on HEp-2 tissue culture cells and chicken intestinal epithelium. Infect Immun. Vol. 74, No. 6, pp. 3156-69.

Ly, K. T. \& Casanova, J. E. (2009). Abelson tyrosine kinase facilitates Salmonella enterica serovar Typhimurium entry into epithelial cells. Infect Immun. Vol. 77, No. 1, pp. 60-9.

Mammarappallil, J. G. \& Elsinghorst, E. A. (2000). Epithelial cell adherence mediated by the enterotoxigenic Escherichia coli tia protein. Infect Immun. Vol. 68, No. 12, pp. 6595-601.

Mattei, P. J., Faudry, E., Job, V., Izore, T., Attree, I. \& Dessen, A. (2011). Membrane targeting and pore formation by the type III secretion system translocon. FEBS J. Vol. 278, No. 3, pp. 414-26.

McGhie, E. J., Hayward, R. D. \& Koronakis, V. (2001). Cooperation between actin-binding proteins of invasive Salmonella: SipA potentiates SipC nucleation and bundling of actin. EMBO J. Vol. 20, No. 9, pp. 2131-9.

McGhie, E. J., Hayward, R. D. \& Koronakis, V. (2004). Control of actin turnover by a Salmonella invasion protein. Mol Cell. Vol. 13, No. 4, pp. 497-510.

Mecsas, J., Welch, R., Erickson, J. W. \& Gross, C. A. (1995). Identification and characterization of an outer membrane protein, OmpX, in Escherichia coli that is homologous to a family of outer membrane proteins including Ail of Yersinia enterocolitica. J Bacteriol. Vol. 177, No. 3, pp. 799-804.

Michael, B., Smith, J. N., Swift, S., Heffron, F. \& Ahmer, B. M. (2001). SdiA of Salmonella enterica is a LuxR homolog that detects mixed microbial communities. J Bacteriol. Vol. 183, No. 19, pp. 5733-42.

Mijouin, L., Rosselin, M., Bottreau, E., Pizarro-Cerda, J., Cossart, P., Velge, P. \& Wiedemann A (2012). /Salmonella/ Enteritidis Rck-mediated invasion requires activation of Rac1 dependent of Class I PI 3-kinases-Akt signalling pathway. /FASEB J./ vol.26, No.4, pp 1569-81.

Miki, T., Okada, N., Shimada, Y. \& Danbara, H. (2004). Characterization of Salmonella pathogenicity island 1 type III secretion-dependent hemolytic activity in Salmonella enterica serovar Typhimurium. Microb Pathog. Vol. 37, No. 2, pp. 65-72.

Miller, S. I., Kukral, A. M. \& Mekalanos, J. J. (1989). A two-component regulatory system (phoP phoQ) controls Salmonella typhimurium virulence. Proc Natl Acad Sci U S A. Vol. 86, No. 13, pp. 5054-8.

Miller, V. L., Beer, K. B., Heusipp, G., Young, B. M. \& Wachtel, M. R. (2001). Identification of regions of Ail required for the invasion and serum resistance phenotypes. Mol Microbiol. Vol. 41, No. 5, pp. 1053-62.

Misselwitz, B., Dilling, S., Vonaesch, P., Sacher, R., Snijder, B., Schlumberger, M., Rout, S., Stark, M., von Mering, C., Pelkmans, L. \& Hardt, W. D. (2011). RNAi screen of Salmonella invasion shows role of COPI in membrane targeting of cholesterol and Cdc42. Mol Syst Biol. Vol. 7, No., pp. 474. 
Mullins, R. D., Heuser, J. A. \& Pollard, T. D. (1998). The interaction of Arp2/3 complex with actin: nucleation, high affinity pointed end capping, and formation of branching networks of filaments. Proc Natl Acad Sci U S A. Vol. 95, No. 11, pp. 6181-6.

Myeni, S. K. \& Zhou, D. (2010). The C terminus of SipC binds and bundles F-actin to promote Salmonella invasion. J Biol Chem. Vol. 285, No. 18, pp. 13357-63.

Nichols, C. D. \& Casanova, J. E. (2010). Salmonella-directed recruitment of new membrane to invasion foci via the host exocyst complex. Curr Biol. Vol. 20, No. 14, pp. 1316-20.

Norris, F. A., Wilson, M. P., Wallis, T. S., Galyov, E. E. \& Majerus, P. W. (1998). SopB, a protein required for virulence of Salmonella dublin, is an inositol phosphate phosphatase. Proc Natl Acad Sci U S A. Vol. 95, No. 24, pp. 14057-9.

Oliveira, M. J., Costa, A. C., Costa, A. M., Henriques, L., Suriano, G., Atherton, J. C., Machado, J. C., Carneiro, F., Seruca, R., Mareel, M., Leroy, A. \& Figueiredo, C. (2006). Helicobacter pylori induces gastric epithelial cell invasion in a c-Met and type IV secretion system-dependent manner. J Biol Chem. Vol. 281, No. 46, pp. 34888-96.

Patel, J. C. \& Galan, J. E. (2005). Manipulation of the host actin cytoskeleton by Salmonella-all in the name of entry. Curr Opin Microbiol. Vol. 8, No. 1, pp. 10-5.

Patel, J. C. \& Galan, J. E. (2006). Differential activation and function of Rho GTPases during Salmonella-host cell interactions. J Cell Biol. Vol. 175, No. 3, pp. 453-63.

Penheiter, K. L., Mathur, N., Giles, D., Fahlen, T. \& Jones, B. D. (1997). Non-invasive Salmonella typhimurium mutants are avirulent because of an inability to enter and destroy M cells of ileal Peyer's patches. Mol Microbiol. Vol. 24, No. 4, pp. 697-709.

Perrett, C. A. \& Jepson, M. A. (2009). Regulation of Salmonella-induced membrane ruffling by SipA differs in strains lacking other effectors. Cell Microbiol. Vol. 11, No. 3, pp. 475-87.

Pollard, T. D., Blanchoin, L. \& Mullins, R. D. (2000). Molecular mechanisms controlling actin filament dynamics in nonmuscle cells. Annu Rev Biophys Biomol Struct. Vol. 29, No., pp. 545-76.

Queiroz, M. H., Madrid, C., Paytubi, S., Balsalobre, C. \& Juarez, A. (2011). Integration Host Factor alleviates H-NS silencing of the Salmonella enterica serovar Typhimurium master regulator of SPI1, hilA. Microbiology. Vol. No.

Radtke, A. L., Wilson, J. W., Sarker, S. \& Nickerson, C. A. (2010). Analysis of interactions of Salmonella type three secretion mutants with 3-D intestinal epithelial cells. PLoS One. Vol. 5, No. 12, pp. e15750.

Rathinavelan, T., Tang, C. \& De Guzman, R. N. (2011). Characterization of the interaction between the Salmonella type III secretion system tip protein SipD and the needle protein PrgI by paramagnetic relaxation enhancement. J Biol Chem. Vol. 286, No. 6, pp. 4922-30.

Reeve, J. N. \& Shaw, J. E. (1979). Lambda encodes an outer membrane protein: the lom gene. Mol Gen Genet. Vol. 172, No. 3, pp. 243-8.

Rittmeyer, E. N., Daniel, S., Hsu, S. C. \& Osman, M. A. (2008). A dual role for IQGAP1 in regulating exocytosis. J Cell Sci. Vol. 121, No. Pt 3, pp. 391-403.

Rosselin, M., Abed, N., Virlogeux-Payant, I., Bottreau, E., Sizaret, P. Y., Velge, P. \& Wiedemann, A. (2011). Heterogeneity of type III secretion system (T3SS)-1independent entry mechanisms used by Salmonella Enteritidis to invade different cell types. Microbiology. Vol. 157, No. Pt 3, pp. 839-47.

Rosselin, M., Virlogeux-Payant, I., Roy, C., Bottreau, E., Sizaret, P. Y., Mijouin, L., Germon, P., Caron, E., Velge, P. \& Wiedemann, A. (2010). Rck of Salmonella enterica, subspecies enterica serovar enteritidis, mediates zipper-like internalization. Cell Res. Vol. 20, No. 6, pp. 647-64. 
Rotger, R. \& Casadesus, J. (1999). The virulence plasmids of Salmonella. Int Microbiol. Vol. 2, No. 3, pp. 177-84.

Samudrala, R., Heffron, F. \& McDermott, J. E. (2009). Accurate prediction of secreted substrates and identification of a conserved putative secretion signal for type III secretion systems. PLoS Pathog. Vol. 5, No. 4, pp. e1000375.

Scherer, C. A., Cooper, E. \& Miller, S. I. (2000). The Salmonella type III secretion translocon protein SspC is inserted into the epithelial cell plasma membrane upon infection. Mol Microbiol. Vol. 37, No. 5, pp. 1133-45.

Schlumberger, M. C., Friebel, A., Buchwald, G., Scheffzek, K., Wittinghofer, A. \& Hardt, W. D. (2003). Amino acids of the bacterial toxin SopE involved in G nucleotide exchange on Cdc42. J Biol Chem. Vol. 278, No. 29, pp. 27149-59.

Schraidt, O. \& Marlovits, T. C. (2011). Three-dimensional model of Salmonella's needle complex at subnanometer resolution. Science. Vol. 331, No. 6021, pp. 1192-5.

Schroeder, G. N. \& Hilbi, H. (2008). Molecular pathogenesis of Shigella spp.: controlling host cell signaling, invasion, and death by type III secretion. Clin Microbiol Rev. Vol. 21, No. 1, pp. 134-56.

Sechi, A. S. \& Wehland, J. (2000). The actin cytoskeleton and plasma membrane connection: PtdIns $(4,5) \mathrm{P}(2)$ influences cytoskeletal protein activity at the plasma membrane. $J$ Cell Sci. Vol. 113 Pt 21, No., pp. 3685-95.

Shi, J. \& Casanova, J. E. (2006). Invasion of host cells by Salmonella typhimurium requires focal adhesion kinase and p130Cas. Mol Biol Cell. Vol. 17, No. 11, pp. 4698-708.

Smith, J. N. \& Ahmer, B. M. (2003). Detection of other microbial species by Salmonella: expression of the SdiA regulon. J Bacteriol. Vol. 185, No. 4, pp. 1357-66.

Smith, J. N., Dyszel, J. L., Soares, J. A., Ellermeier, C. D., Altier, C., Lawhon, S. D., Adams, L. G., Konjufca, V., Curtiss, R., 3rd, Slauch, J. M. \& Ahmer, B. M. (2008). SdiA, an $\mathrm{N}$-acylhomoserine lactone receptor, becomes active during the transit of Salmonella enterica through the gastrointestinal tract of turtles. PLoS One. Vol. 3, No. 7, pp. e2826.

Stebbins, C. E. \& Galan, J. E. (2000). Modulation of host signaling by a bacterial mimic: structure of the Salmonella effector SptP bound to Rac1. Mol Cell. Vol. 6, No. 6, pp. 1449-60.

Takeuchi, A. (1967). Electron microscope studies of experimental Salmonella infection. I. Penetration into the intestinal epithelium by Salmonella typhimurium. Am J Pathol. Vol. 50, No. 1, pp. 109-36.

Tampakaki, A. P., Fadouloglou, V. E., Gazi, A. D., Panopoulos, N. J. \& Kokkinidis, M. (2004). Conserved features of type III secretion. Cell Microbiol. Vol. 6, No. 9, pp. 805-16.

Terebiznik, M. R., Vieira, O. V., Marcus, S. L., Slade, A., Yip, C. M., Trimble, W. S., Meyer, T., Finlay, B. B. \& Grinstein, S. (2002). Elimination of host cell PtdIns $(4,5) P(2)$ by bacterial SigD promotes membrane fission during invasion by Salmonella. Nat Cell Biol. Vol. 4, No. 10, pp. 766-73.

van Sorge, A. J., Termote, J. U., de Vries, M. J., Boonstra, F. N., Stellingwerf, C. \& SchalijDelfos, N. E. (2011). The incidence of visual impairment due to retinopathy of prematurity (ROP) and concomitant disabilities in the Netherlands: a 30 year overview. Br J Ophthalmol. Vol. 95, No. 7, pp. 937-41.

Vazquez-Torres, A., Jones-Carson, J., Baumler, A. J., Falkow, S., Valdivia, R., Brown, W., Le, M., Berggren, R., Parks, W. T. \& Fang, F. C. (1999). Extraintestinal dissemination of Salmonella by CD18-expressing phagocytes. Nature. Vol. 401, No. 6755, pp. 804-8. 
Velge, P., Cloeckaert, A. \& Barrow, P. (2005). Emergence of Salmonella epidemics: the problems related to Salmonella enterica serotype Enteritidis and multiple antibiotic resistance in other major serotypes. Vet Res. Vol. 36, No. 3, pp. 267-88.

Vivero, A., Banos, R. C., Mariscotti, J. F., Oliveros, J. C., Garcia-del Portillo, F., Juarez, A. \& Madrid, C. (2008). Modulation of horizontally acquired genes by the Hha-YdgT proteins in Salmonella enterica serovar Typhimurium. J Bacteriol. Vol. 190, No. 3, pp. 1152-6.

Wai, S. N., Lindmark, B., Soderblom, T., Takade, A., Westermark, M., Oscarsson, J., Jass, J., Richter-Dahlfors, A., Mizunoe, Y. \& Uhlin, B. E. (2003). Vesicle-mediated export and assembly of pore-forming oligomers of the enterobacterial ClyA cytotoxin. Cell. Vol. 115, No. 1, pp. 25-35.

Wallar, L. E., Bysice, A. M. \& Coombes, B. K. (2011). The non-motile phenotype of Salmonella hha ydgT mutants is mediated through PefI-SrgD. BMC Microbiol. Vol. 11, No., pp. 141.

Wallis, T. S. \& Galyov, E. E. (2000). Molecular basis of Salmonella-induced enteritis. Mol Microbiol. Vol. 36, No. 5, pp. 997-1005.

Watson, K. G. \& Holden, D. W. (2010). Dynamics of growth and dissemination of Salmonella in vivo. Cell Microbiol. Vol. 12, No. 10, pp. 1389-97.

Winnen, B., Schlumberger, M. C., Sturm, A., Schupbach, K., Siebenmann, S., Jenny, P. \& Hardt, W. D. (2008). Hierarchical effector protein transport by the Salmonella Typhimurium SPI-1 type III secretion system. PLoS One. Vol. 3, No. 5, pp. e2178.

Wozniak, C. E., Lee, C. \& Hughes, K. T. (2009). T-POP array identifies EcnR and PefI-SrgD as novel regulators of flagellar gene expression. J Bacteriol. Vol. 191, No. 5, pp. 1498-508.

Yao, Y., Dickerson, T. J., Hixon, M. S. \& Dyson, H. J. (2007). NMR detection of adventitious xylose binding to the quorum-sensing protein SdiA of Escherichia coli. Bioorg Med Chem Lett. Vol. 17, No. 22, pp. 6202-5.

Zhou, D., Chen, L. M., Hernandez, L., Shears, S. B. \& Galan, J. E. (2001). A Salmonella inositol polyphosphatase acts in conjunction with other bacterial effectors to promote host cell actin cytoskeleton rearrangements and bacterial internalization. Mol Microbiol. Vol. 39, No. 2, pp. 248-59.

Zhou, D., Mooseker, M. S. \& Galan, J. E. (1999a). An invasion-associated Salmonella protein modulates the actin-bundling activity of plastin. Proc Natl Acad Sci U S A. Vol. 96, No. 18, pp. 10176-81.

Zhou, D., Mooseker, M. S. \& Galan, J. E. (1999b). Role of the S. typhimurium actin-binding protein SipA in bacterial internalization. Science. Vol. 283, No. 5410, pp. 2092-5. 
(C) 2012 The Author(s). Licensee IntechOpen. This is an open access article distributed under the terms of the Creative Commons Attribution 3.0 License, which permits unrestricted use, distribution, and reproduction in any medium, provided the original work is properly cited. 